\title{
Fire raiser or fire accelerant? A meeting report on the 14th International TNF Conference 2013
}

\author{
S von Karstedt ${ }^{1,2}$ and N Peltzer ${ }^{1,2}$
}

Cell Death and Differentiation (2014) 21, 1343-1344; doi:10.1038/cdd.2014.43; published online 11 April 2014

Meeting report on the 14th International TNF Conference. Loews Le Concorde, Quebec City, Quebec, Canada, 7-10 July 2013

The 14th TNF conference, held in the city of Quebec, Canada, provided a comprehensive platform to discuss exciting novel findings that will significantly advance the field in years to come. Organized by Jen Gommerman, Linda Burkly, Gail Bishop, Carl Ware and Tania Watts, the program comprised an excellent choice of talks and posters providing novel insight into signaling pathways of tumor necrosis factor (TNF)superfamily members triggering autoimmune inflammation, cell death and infection-related immune response. In particular, the questions whether non-apoptotic cell death would cause autoimmune inflammation and therefore precede it, whether cell death was a consequence of aberrant inflammation and whether players of the non-apoptotic cell death machinery had a 'day job' in promoting inflammation independently of cell death induction were intensely debated. In the following meeting report, we will summarize data and ideas discussed at the meeting. Owing to space limitations, we have focused on summarizing scientific findings that dealt with the above-mentioned topics and unpublished data.

Nancy Ruddle gave a history of the discovery of the founding members of the TNF-superfamily lymphotoxin $\alpha$ $(\mathrm{LT} \alpha)$ and TNF, and the contributions of Lloyd Old in characterizing them. Sarah Hymowitz showed that LT $\beta R$ required heterotrimeric $\mathrm{LT} \alpha_{1} \beta_{2}$ binding and in particular the $\mathrm{LT} \beta$-LT $\alpha$ interface to fully activate the lymphotoxin signaling pathway. Mathias Heikenwälder presented exciting data on the generation of mice overexpressing $L T \alpha / \beta$ from the Elastase promoter that developed symptoms mimicking human autoimmune pancreatitis such as nuclear p65, high levels of pStat3 and autoantibody production. This talk was followed by Sergei Nedaspasov, who reported on his finding that ROR $\gamma$ T-cell-specific LT $\alpha$ knockout mice lack lymph nodes and Peyer's patches, and present decreased fecal levels of $\lg A$ suggesting that production of $L T \alpha$ by $T$ cells is important for intestinal immunity. David Wallach showed that inflammatory genes such as IL-1 $\beta$ were upregulated before birth in the skin of keratinocyte-specific caspase-8-deficient mice in the absence of detectable amounts of cell death. Further supporting a role of caspase- 8 in the inhibition of inflammation without affecting non-apoptotic cell death, he demonstrated that caspase-8-deficient dendritic cells showed increased formation of the inflammasome and RIPK1 kinase activityand RIPK3-dependent IL-1 $\beta$ processing upon LPS stimulation. Again, no significant amounts of cell death could be detected, highlighting a function of RIPK1 and RIPK3 in regulating the inflammasome.

Hao Wu presented data showing that proteins of the CARMA1-BCL10-MALT1 complex downstream of TCR signaling assemble in filaments. In these filaments, CARMA1 nucleated the helical polymerization of BCL10 inducing activation conformation in MALT1. Picking up discussions of the night before, Nieves Peltzer from the group of Henning Walczak reported that mice deficient for HOIP, the central component of the linear ubiquitin chain assembly complex (LUBAC), are embryonically lethal. Lethality occurred at E10.5 and was associated with increased cell death in the yolk sac and heart, leading to cardiovascular defects. Manolis Pasparakis reported that keratinocyte-specific deletion of TNFR1 but not RIPK3 rescued dermatitis of mice deficient in the LUBAC component SHARPIN (cpdm-mice). Combined deletion of RIPK3 and FADD in keratinocytes was also sufficient to rescue dermatitis of $c p d m$-mice, indicating that this is most likely caused by apoptotic and/or necroptotic cell death. John Silke presented that macrophages are the source of TNF that causes cpdm-dermatitis. In slight contrast to the Pasparakis' group, he showed that absence of RIPK3, and also MLKL, a downstream target of RIPK3 in the necroptosis pathway, provided partial, and the RIPK3 ${ }^{-/-} /$caspase- $8^{-1+}$ combination complete protection from $c p d m$-dermatitis. Surprisingly, however, RIPK3/caspase-8 double knockout on a $c p d m$ background resulted in embryonic lethality. These data raise the interesting question of the nature of the death pathway (if indeed the lethality is due to exacerbated cell

\footnotetext{
${ }^{1}$ Centre for Cell Death, Cancer and Inflammation, UCL Cancer Institute, London, UK ${ }^{*}$ Corresponding author: S von Karstedt, Centre for Cell Death, Cancer and Inflammation, UCL Cancer Institute, 72 Huntley Street, London WC1E 6DD, UK. Tel: +44 207679 46804; Fax: +44 207679 6925; E-mail: s.karstedt@ucl.ac.uk

${ }^{2}$ These authors contributed equally to this work.
} 
death) that occurs in the absence of SHARPIN, RIPK3 and caspase-8.

Connecting this question with the point that RIPK3 might have a day job in regulating inflammation, Monica Yabal showed that TNF treatment of XIAP KO MEFs led to markedly enhanced IL $1-\beta$ secretion that was accompanied by caspase-8 activation, enhanced inflammasome formation and increased cell death. Strikingly, all of these effects were neutralized by the absence of RIPK3 or blocked by necrostatin-1, possibly placing RIPK3 and RIPK1 also at the crossroads of inflammasome-mediated cell death and IL1- $\beta$ maturation.

Further investigating the role of RIPKs in inflammatory processes, Francis Chan presented data showing that RIPK3 knockout animals were unable to recover from DSS-induced colitis due to defective non-canonical NF- $\kappa \mathrm{B}$ activation and IL22 production by dendritic cells. The importance of RIPK3 in DSS colitis recovery was also shown by Peter Vandenabeele and he extended this line of investigation by showing that RIPK3 was also required for colorectal cancer development induced by DSS + AOM. William Kaiser again looked into the death-promoting role of RIPK3, demonstrating that perinatal lethality of RIPK1-knockout animals could be rescued by combined absence of RIPK3 and caspase-8. Interestingly, $\mathrm{RIPK}^{-/-} /$Caspase- $8^{-/-} / \mathrm{RIPK}^{+/-}$mice were viable and did not show lymphadenopathy as seen in RIPK3/Caspase-8 double knockout animals.

Domagoj Vucic addressed a more molecular perspective on cell death induction by TNF-superfamily members by showing that sensitivity to smac mimetics in cell lines is associated with markedly increased RIPK1 ubiquitination. He also showed that the deubiquitinase OTUB1 acts as a negative regulator of TWEAK-induced cell death by removing K48-linked ubiquitin chains from clAP1.

Gail Bishop showed that TRAF3-deficient T-cells produced less chemo/cytokines upon TCR stimulation due to impaired Fyn and Lck phosphorylation. Lauren Workman reported on an important role of TRAF2 phosphorylation by MEKK1 on Ser-11 for full JNK and NF- $\kappa$ B activation by CD40 and suggested that B-cell-derived neoplasms might use high TRAF2 phosphorylation for elevated NF- $\kappa$ B activation and apoptosis resistance. Another important phosphorylation of a TRAF was reported by Laurent Sabbagh, who showed that TRAF1 Ser-139 phosphorylation was required to limit the duration of non-canonical NF- $\kappa \mathrm{B}$ activation triggered by 4-1BB on CD8 $T$ cells.

Describing an unexpected role for endogenous TRAIL-R2, Silvia von Karstedt showed that KRAS/p53-mutation-driven pancreatic cancer requires constitutive endogenous TRAILR2 stimulation for cancer cell-autonomous migration and metastasis in vivo. Importantly, absence of murine TRAIL-R from tumor cells markedly increased survival of KRAS/p53 double-mutant mice. These data strongly suggest considering blockage of endogenous TRAIL and/or TRAIL-R2 in KRASmutated cancers. Pamela Holland showed that combined treatment with a TRAIL-R2-specific agonistic antibody and soluble untagged TRAIL achieved synergistic induction of apoptosis via enhanced TRAIL-R2 crosslinking, whereas none of the two clinically available TRAIL-R agonists alone was capable of doing so. Chris Benedict presented that the human CMV viral protein UL141 prevents host cell apoptosis by sequestering TRAIL-R2 in the ER, demonstrating an elegant mechanism of pathogen-host co-evolution.

After three intense days, the 14th International TNF Conference drew to a close with many additional interesting contributions presented as either talks or posters that will be covered in more detail in an edition of Cytokine and Growth Factors. The next TNF meeting will take place in Ghent, Belgium 2015 and as it will be another francophone meeting: A bientôt!

\section{Conflict of Interest}

The authors declare no conflict of interest.

Acknowledgements. We thank John Silke for the helpful editing of the report. This meeting was financially supported by Biogen Idec, UCB, Medlmmune, EMD, Arthritis National Research Foundation, Genentech, abbvie, AdipoGen, AMGEN, Janssen, Pfizer, The Jackson Laboratory, DeBioPharm Group, Novartis, Kyowa Kirin, Becton Dickinson, BioLegend, Cell Death \& Differentiation, Edimer, and Georges Chappuis and Diane and Elliot Feuerstein of the Jewish Community Foundation. 